\title{
Transitional cell carcinoma arising in a calyceal cyst mimicking a cystic renal tumour
}

\author{
Jeong Ho Kim, MD; ${ }^{*}$ Joo Yeon Song, MD; ${ }^{\dagger}$ Wan Lee, $M D^{*}$
}

*Department of Urology, Dongnam Institution of Radiological \& Medical Sciences Cancer Center, Korea; 'Department of Pathology, Dongnam Institution of Radiological \& Medical Sciences Cancer Center, Korea

Cite as: Can Urol Assoc J 2014;8(3-4):e196-8. http://dx.doi.org/10.5489/cuaj.1528 Published online March 11, 2014.

\section{Abstract}

Solitary renal cysts are relatively common. The occurrence of transitional cell carcinoma (TCC) in a renal cyst is rare. We present the case of a 59-year-old man with a medical history of viral hepatitis B. During a workup for his hepatitis, a computed tomography scan revealed a large cystic tumour in the upper region of the left kidney. A radical left nephrectomy was performed. Microscopic examination of the cystic tumour revealed a grade 2 TCC. The cyst was lined by transitional epithelium. This is a case of a TCC growing within a renal calyceal cyst.

\section{Introduction}

Renal cysts are associated with different types of kidney diseases, and some cysts are difficult to distinguish in imaging. Particularly, a calyceal cyst, which is very rare, is difficult to diagnose because of its similarity to renal cysts. Since cases of calyceal cyst are extremely rare, most renal cysts are likely to be seen in the renal parenchyma. We report the case of a patient who was initially diagnosed with cystic renal tumour. This case is unique in that the initial diagnosis was eventually confirmed as transitional cell carcinoma (TCC) from the biopsy.

\section{Case report}

A 59-year-old man, a hepatitis B carrier, presented for treatment of a left renal cyst detected on abdominal computed tomography $(\mathrm{CT})$. The patient did not present with gross hematuria or other urinary symptoms. No abnormal findings were detected in the physical examination and no left costovertebral angle tenderness. All blood and urine tests were normal. Abdominal CT revealed 1 cyst, measuring $8.7 \mathrm{~cm}$ in diameter in the upper left kidney and another small cyst in the right kidney (Fig. 1, part A).
Calcification was present on the left renal cyst wall, and a contrast-enhanced mass was found on the bottom of the cyst (Fig. 1, part B). However, identification of the exact shape was difficult because of the lack of a coronal image. The renal cyst had enlarged in size compared to its size 5 months before. Lymphadenopathy was absent, and there were no abnormal findings observed on the ureter or the bladder. To obtain more information about the contrast-enhanced mass, we conducted a Doppler ultrasound examination on the kidney; a $3.9 \times 2.4-\mathrm{cm}$ solid mass with angiogenesis was observed in the lower left kidney. The cyst was separated into several septa with multiple nodules each (Fig. 2).

The patient underwent a left laparoscopic radical nephrectomy. Since the large tumour prevented visualization of a clear operative field, resection of the renal hilum was difficult. Slight bleeding occurred during resection because of the severe adhesion of the tumour with the psoas major muscle and the spleen. The resection was completed carefully, and the tumour was successfully removed without rupturing. The ureter was resected after ligation in the upper ureter. After the surgery, the patient recovered uneventfully and was discharged on postoperative day 8 . A cystic tumour measuring $10.0 \times 8.7 \times 7.8 \mathrm{~cm}$ was found on pathological examination. Many nodules were observed in the septa within the tumour, and a light yellow $4.0 \times 2.5-\mathrm{cm}$ mass was found at the bottom of the tumour (Fig. 3).

Microscopic examination revealed that the tumour wall was composed of urothelium. The interior mass was a grade 2/3 TCC (Fig. 4).

Although the cancer had invaded the muscle layers, no invasion of the tissues surrounding the renal medulla and renal pelvis was noted. Although there was no direct communication between the tumour and the renal pelvis, the tumour had partially invaded the renal pelvis and proximal ureter. The tumour was cystic at the renal calyx because it was clearly differentiable from the renal pelvis and exerted no pressure upon it. Since the patient was diagnosed with TNM stage T2NOM0, we decided to monitor him without implementing adjuvant chemotherapy. The patient remains free of disease 10 months after surgery. 


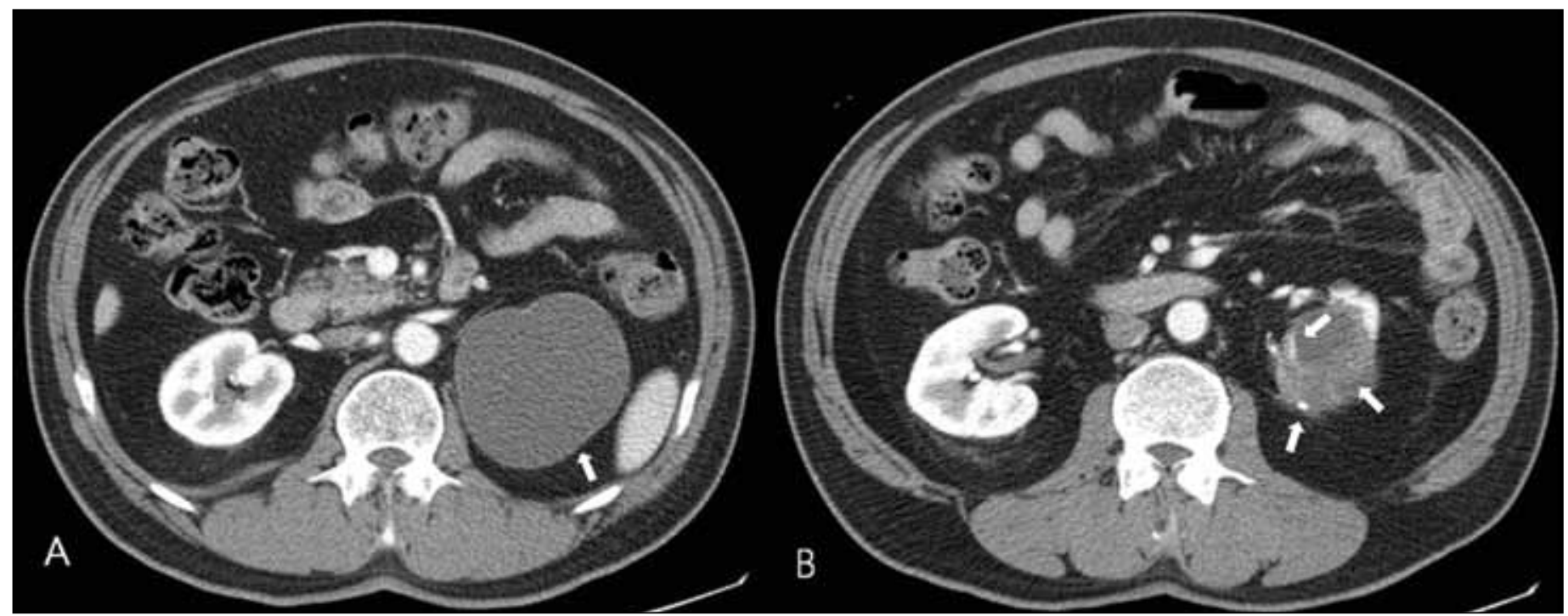

Fig. 1. A. A computed tomography (CT) scan shows a large cystic tumour in the upper pole of the left kidney. B. Contrast-enhanced CT scan shows the bottom of the cyst which has enhanced wall and small amount of calcification. Slightly enhanced mass is seen.

\section{Discussion}

Simple cysts are commonly found in the kidney and do not require treatment in most cases. Bosniak published a renal cyst classification system based on shape; it is widely used. McFarlane and colleagues analyzed the existing literature and reported the frequency of malignant renal cysts according to Bosniak's classification. ${ }^{2}$ Renal cell carcinoma (RCC) was found in $1.7 \%, 18.5 \%, 33 \%$, and $92.5 \%$ of Bosniak categories I, II, III, and IV, respectively. ${ }^{2}$ Bosniak category IV, which is very likely to be malignant, is defined as the presence of a contrast-enhanced mass within a cyst. ${ }^{1}$ Our case was classified as Bosniak category IV due to the presence of a contrast-enhanced mass in the cyst with a number of nodules on the cyst wall. Since about $85 \%$ of renal malig-

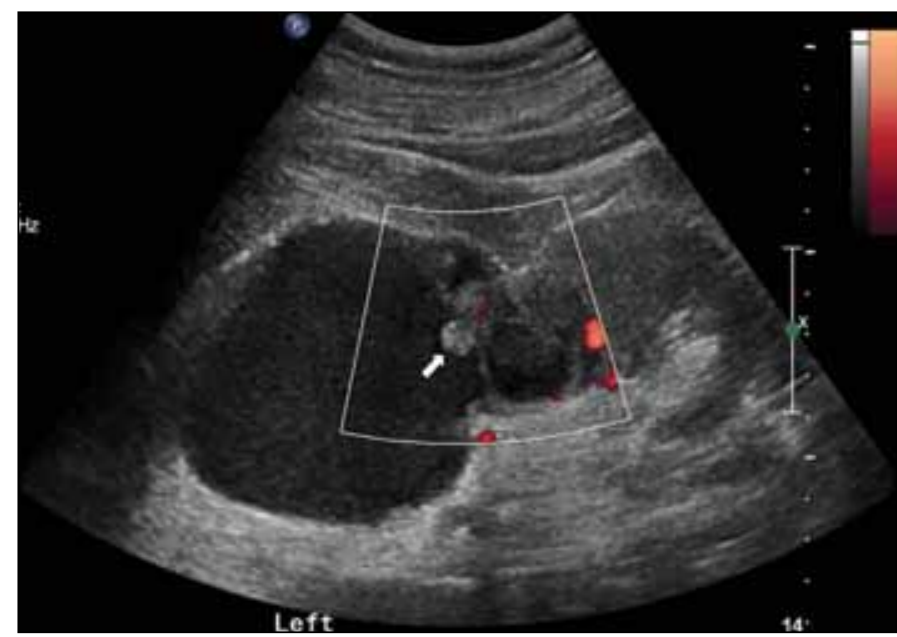

Fig. 2. Ultrasonogram of the left kidney shows multiple masses in the lower portion of the cystic tumour. nant tumours are RCCs, this case was also suspected to be a Bosniak category IV cystic RCC.

Renal cysts occur in the diverticulum of the collecting tubule. Although the causes of renal calyceal cysts have not yet been clearly established, few studies have reported the possibility of congenital or acquired renal calyceal diverticula progressing to renal calyceal cysts. ${ }^{3-5}$ However, the study of the natural course of renal calyceal cysts is expected to be difficult, since renal calyceal diverticula themselves occur infrequently.

The basic treatment for TCC occurring in the upper urinary tract (UUT) consists of radical nephroureterectomy (RNU) with excision of the bladder cuff. ${ }^{6}$ In the present case, the tumour was suspected to be a cystic RCC; there-

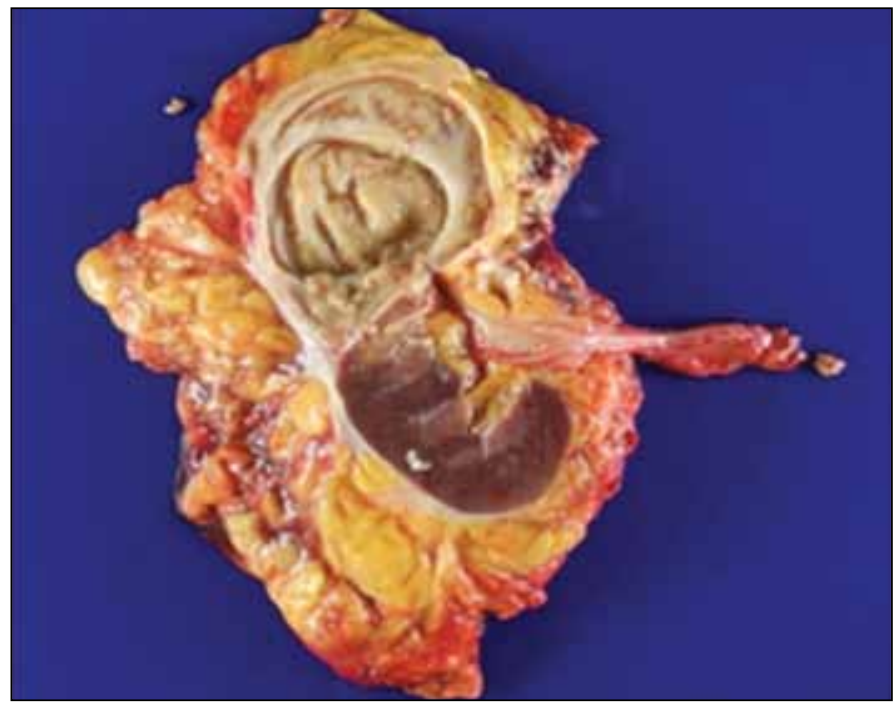

Fig. 3. Coronal section of the left kidney shows a large cystic tumour with multiple papillary solid mural nodules. 
Kim et al.

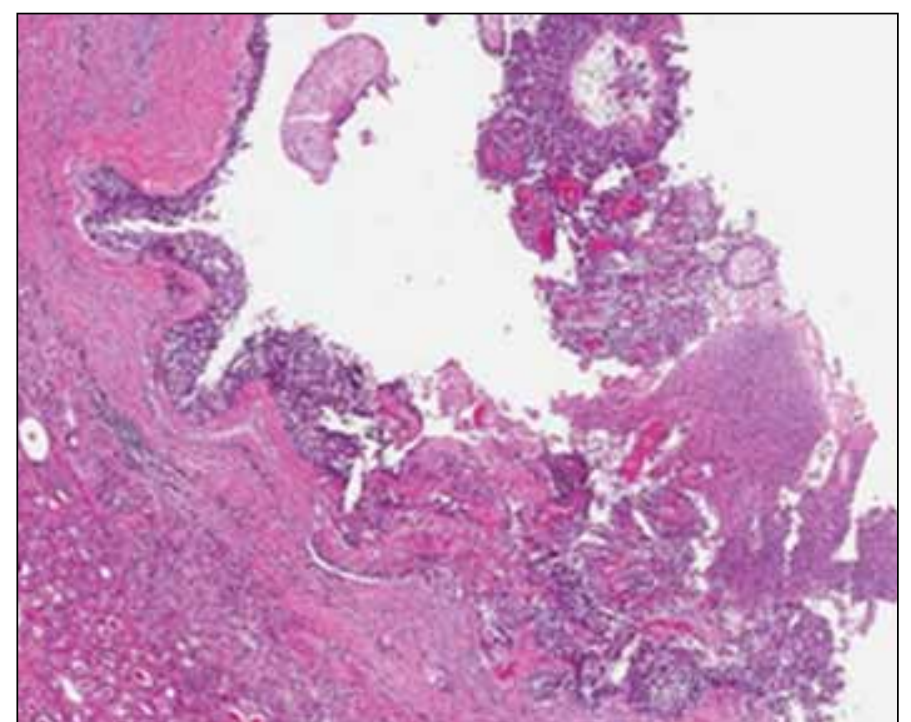

Fig. 4. Mural nodules show grade 2 papillary urothelial carcinoma (right upper). The cystic wall was also lined with carcinoma cells, which focally invades subepithelial connective tissue (left lower) and muscularis (not shown).

fore, the ureter was not completely removed. If the tumour was suspected to be a TCC before surgery, the entire ureter would have been removed. Although part of the ureter remained, the surgical margin was negative. Because no differences were found in recurrence rates in several reports that compared patients who underwent partial ureterectomy and RNU for TCC of the UUT, ${ }^{7,8}$ the patient was monitored without additional surgical treatment. Several evaluations, including cystoscopy, urine cytology and CT, will be performed on a regular basis.

Friedman and colleagues discovered a TCC on CT-guided fine needle aspiration (FNA) biopsy conducted before surgery and subsequently performed a nephroureterectomy. ${ }^{9}$ Roberts and colleagues also performed nephroureterectomy of the rest of the ureter when TCC was identified in a frozen section tissue biopsy conducted during surgery. ${ }^{10}$ In the present case, the patient's condition was diagnosed as cystic RCC, on the basis of pre-surgical CT scans. Since preoperative renal biopsy is not mandatory for tumours in the kidney, a renal biopsy was not performed. However, according to the latest data, percutaneous FNA cytology and percutaneous biopsy had a sensitivity for malignancy detection of $90 \%$, a positive predictive value for cancer of $96 \%$, and a negative predictive value of $80 \% .{ }^{11}$ Moreover, according to a report by Lang and colleagues, suitable tissue for diagnosis could be obtained from $90 \%$ of cases by performing CT-guided biopsy on renal cysts. ${ }^{12}$ In the current case, this procedure might have been helpful in diagnosing the condition, had ultrasonography- or CT-guided biopsy been available prior to surgery. Therefore, the treatment of cystic renal tumours, with a high probability of malignancy, requires the use of various methods to collect sufficient information on the tumour.

\section{Conclusion}

The frequency for the occurrence of calyceal cyst in the kidney is very low. Thus, most cystic renal tumours are considered to occur in the renal parenchyma, and treatments are administered accordingly. However, as demonstrated in rare cases, it is possible that such cysts could be calyceal or peripelvic. Therefore, in the treatment of cystic renal tumours, it is important to check the tumour type through biopsy or FNA cytology.

Competing interests: Dr. Kim, Dr. Song and Dr. Lee all declare no competing financial or personal interests.

This paper has been peer-reviewed.

\section{References}

1. Bosniak MA. The current radiological approach to renal cysts. Radiology 1986;158:1-10.

2. Warren KS, McFarlane J. The Bosniak classification of renal cystic masses. BJU Int 2005;95:939-42. http://dx.doi.org/10.1111/i.1464-410X.2005.05442.x

3. Kramer SA, Hoffman AD, Aydin G, et al. Simple renal cysts in children. J Urol 1982;128:1259-61.

4. Nicholas JL. An unusual complication of calyceal diverticulum. Br J Urol 1975;47:370. http://dx.doi.org/10.1111/i.1464-410X.1975.tb03987.x

5. Mosli H, MacDonald P, Schillinger J. Caliceal diverticula developing into simple renal cyst. I Urol 1986;136:658-61.

6. Roupret $M$, Babjuk M, Comperat E, et al. European guidelines on upper tract urothelial carcinomas: 2013 update. Eur Urol 2013;63:1059-71. http://dx.doi.org/10.1016/j.eururo.2013.03.032

7. Bagrodia A, Kuehhas FE, Gayed BA, et al. Comparative analysis of oncologic outcomes of partial ureterectomy vs radical nephroureterectomy in upper tract urothelial carcinoma. Urology 2013;81:972-7. http://dx.doi.org/10.1016/i.urology.2012.12.059

8. Jeldres C, Lughezzani G, Sun M, et al. Segmental ureterectomy can safely be performed in patients with transitional cell carcinoma of the ureter. J Urol 2010;183:1324-9. http://dx.doi.org/10.1016/i.juro.2009.12.018

9. Friedman HD, Nsouli IS, Krauss DJ, et al. Transitional cell carcinoma arising in a pyelocaliceal cyst. Virchows Arch 1999;434:459-62. http://dx.doi.org/10.1007/s004280050367

10. Roberts $\mathrm{CC}$, Collins JM, Lidner TK, et al. Transitional cell carcinoma obstructing a calyceal diverticulum: unusual presentation as a peripheral cystic mass. J Ultrasound Med 1999;18:19-21.

11. Wolf JS Jr. Evaluation and management of solid and cystic renal masses. J Urol 1998;159:1120-33. http://dx.doi.org/10.1016/S0022-5347(01)63529-8

12. Lang EK, Macchia RJ, Gayle B, et al. CT-guided biopsy of indeterminate renal cystic masses (Bosniak 3 and 2F): accuracy and impact on clinical management. Eur Radiol 2002;12:2518-24.

Correspondence: Dr. Wan Lee, Department of Urology, Dongnam Institution of Radiological \& Medical Sciences Cancer Center, Korea; campfire81@gmail.com 